УДК 621.313.2: 621.7 .08

\title{
РАЗРАБОТКА СПОСОБА ПРОГНОЗИРОВАНИЯ ОСТАТОЧНОГО РЕСУРСА ЭЛЕКТРОЩЕТОК ТЯГОВЫХ ЭЛЕКТРОДВИГАТЕЛЕЙ
}

\author{
Харламов Виктор Васильевич1, \\ emoe@omgups.ru
}

\section{Попов Денис Игоревич1,} emoe@omgups.ru

\author{
Байсадыков Марсель Фаритович1, \\ emoe@omgups.ru \\ 1 Омский государственный университет путей сообщения, \\ Россия, 644046, г. Омск, пр. Маркса, 35.
}

\begin{abstract}
Актуальность исследования определяется необходимостью повышения надежности работы подвижного состава (карьерных самосвалов), обеспечивающего добычу угля открытым способом. Такую же актуальность данная проблема имеет в отрасли железнодорожных перевозок - на подвижном составе железных дорог. Безотказность работы подвижного состава определяется надежностью функционирования его отдельных узлов. Как известно из анализа отказов подвижного состава транспортных средств с коллекторными тяговыми электродвигателями, одним из критичных элементов для них является щеточный аппарат. Известные способы прогнозирования не используют в полной мере возможности современных бортовых систем мониторинга режимов работы тяговых электродвигателей. Следовательно, актуальной задачей является повышение точности прогнозирования остаточного ресурса электрощеток за счет учета показателей режимов работы, фииксируемых бортовой системой мониторинга.

Цель: разработка способа прогнозирования остаточного ресурса электрощеток тяговых электродвигателей карьерных самосвалов с учетом показателей режимов работы, получаемых от бортовой системы мониторинга.

объект: электрощетки тяговых электродвигателей карьерных самосвалов.

Методы: проведение математического анализа данных бортовых систем мониторинга, математическое моделирование процесса изнашивания электрощеток, синтез способа прогнозирования остаточного ресурса щеток тягового электродвигателя.

Результаты. Разработан способ прогнозирования остаточного ресурса щеток тяговых электродвигателей, позволяющий учесть показатели режимов работы в условиях реальной эксплуатации. Повышена точность определения остаточного ресурса электрощеток за счет применения в процессе прогнозирования массивов данных, полученных от бортовой системой мониторинга. Разработан алгоритм прогнозирования, который может быть применен в бортовых электронных системах подвижного состава для индикации остаточного ресурса электрощеток.
\end{abstract}

\section{Ключевые слова:}

Карьерный самосвал, тяговый электродвигатель, износ электрощеток, прогнозирование остаточного ресурса, массивы данных, система мониторинга, математическое моделирование.

\section{Введение}

По данным Министерства энергетики РФ за последние десять лет, за исключением 2020 г., наблюдается устойчивый рост добычи угля в Российской федерации (рис. 1) [1]. Значительная часть добычи осуществляется открытым способом на угольных карьеpax с применением автосамосвалов. Согласно Программе развития угольной промышленности до 2035 г. планируется увеличить добычу угля до 485-685 млн т в год [2].

Решение задачи обеспечения бесперебойной добычи угля на угольных карьерах невозможно без контроля технического состояния и осуществления своевременного обслуживания и ремонта карьерных самосвалов. Аналогичная задача стоит для железнодорожного транспорта в рамках обеспечения надежного функционирования подвижного состава (электровозов, тепловозов и др.). Как показывает анализ статистических данных по отказам подвижного состава с коллекторными тяговыми электродвигателями (ТЭД), в обеих названных отраслях промышленности повышенный износ щеток и неисправности, связанные с коллекторно-щеточным узлом наряду с повреждениями подшипниковых узлов и изоляции токопроводящих частей, являются наиболее частыми [3-6] (рис. 2).

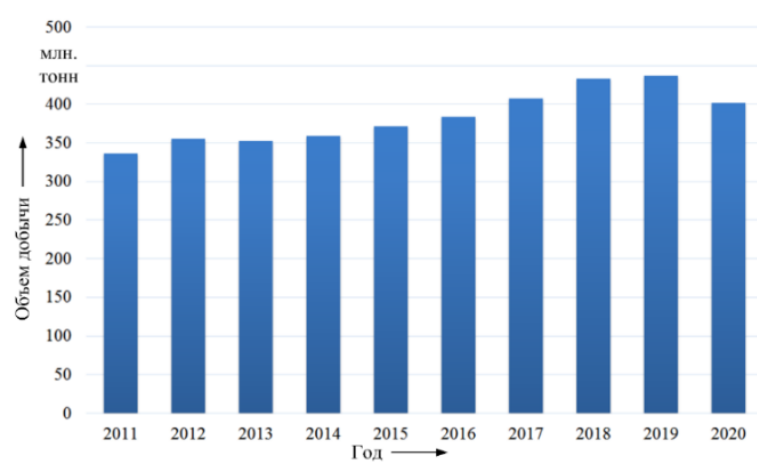

Pис. 1. Объем добычи угля в России в 2011-2020 г2.

Fig. 1. Volume of coal production in Russia in 2011-2020 


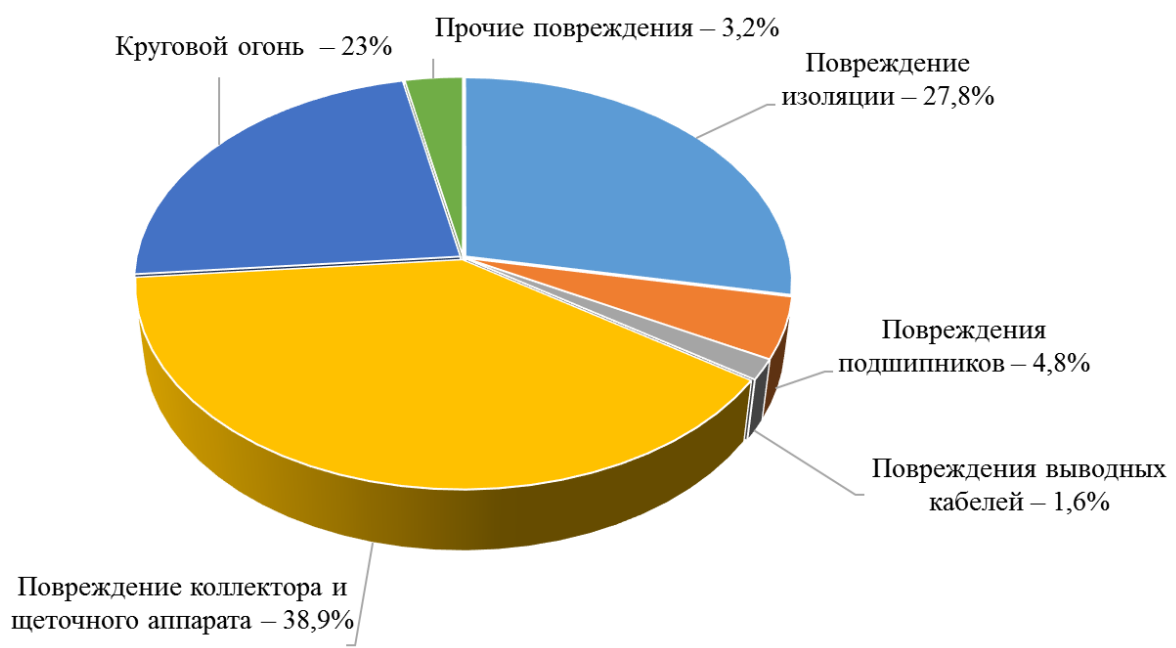

Рис. 2. Статистика отказов ТЭД постоянного тока карьерных самосвалов БелАЗ грузоподъемностью $220 \mathrm{~m}$

Fig. 2. Failure statistics of DC traction motors of BelAZ dump trucks with a carrying capacity of 220 tons

Как показывает анализ статистики отказов коллекторных ТЭД карьерных самосвалов БЕЛАЗ, значительная доля отказов приходится на повреждения коллектора и щеточного аппарата, из которых каждый четвертый приходится непосредственно на щеточный аппарат.

С точки зрения возможной тяжести последствий выхода из строя коллекторно-щеточный узел может быть назван критичным элементом [7].

\section{Текущее состояние разработанности проблемы}

Известны различные способы определения ресурса работы щеток коллекторных электродвигателей, которые сводятся к сравнению текущей $\left(l_{\text {щ }}\right)$ и допустимой высоты щетки $l_{\text {щ. д }}$ и вычислению интенсивности ее изнашивания $\left(\frac{\Delta И}{\Delta t}\right)$ [8-11]. Зная данные величины, можно определить ресурс щеток по выражению:

$$
T_{\text {щ. } \mathrm{p}}=\frac{l_{\text {щ }}-l_{\text {щ.д }}}{\frac{\Delta И}{\Delta t}} .
$$

Математическая модель процесса изнашивания электрощетки представляет собой выражение, в котором интенсивность износа складывается из трех составляющих - фрикционной $\left(\frac{\Delta И_{\phi}}{\Delta t}\right)$, электрокоррозионной $\left(\frac{\Delta И_{\mathrm{\kappa}}}{\Delta t}\right)$ и электроэрозионной $\left(\frac{\Delta И_{\text {эр }}}{\Delta t}\right)$ [11-13]:

$$
\frac{\Delta И}{\Delta t}=K_{\phi} n N_{\text {к. ср }}+K_{\text {к }} \cdot n^{\frac{3}{4}} I_{\text {щ }}^{\frac{1}{2}} N_{\text {к. ср }}+K_{\text {эр }} n P_{\text {и }},
$$

где $K_{\phi}, K_{\mathrm{K}}, K_{\text {эр }}$ - имеющие размерность коэффициенты, определяющие интенсивность соответствующих составляющих износа, зависящие от конструктивных параметров коллекторно-щеточного узла; $n$ - частота вращения якоря, об/мин; $N_{\text {к. ср }}$ среднее нажатие щетки на коллектор, принимаемое равным среднему нажатию рычага щеткодержателя на щетку $N_{\text {p. ср }}, \mathrm{H}$; $I_{\text {щ }}$ - сила тока, проходящего по щетке, $\mathrm{A} ; P_{\text {и }}-$ мощность искрения, о. е.
Различные способы определения ресурса работы электрощеток имеют свои недостатки. Наиболее обоснованным с точки зрения простоты и точности практического определения коэффициентов $K_{\phi}, K_{\mathrm{\kappa}}$, $K_{\text {эр }}$ является способ, приведенный в [12]. В соответствии с данным способом в связи с нелинейной зависимостью электрокорозионной составляющей от силы нажатия $\left(N_{\kappa}\right)$ предложено учитывать колебания данной силы, обусловленные воздействием профиля коллектора, путем математического моделирования движения щетки в щеткодержателе. При описании данного способа в [12] обосновано применение математической модели механического взаимодействия щетки с коллектором в двух степенях свободы, представляющее собой систему из двух дифференциальных уравнений:

$$
\left\{\begin{array}{l}
M \ddot{z}+F_{\text {тшд1 }}(y, \dot{z})+F_{\text {тш } 2}(y, \dot{z})- \\
\quad-N_{\mathrm{p}}-M g+N_{\mathrm{\kappa}}(y, z, \eta)=0 \\
M \ddot{y}+F_{\text {трр }}\left(N_{\mathrm{p}}, \dot{y}\right)+F_{\text {трк }}\left(N_{\mathrm{\kappa}}, \dot{y}\right)-Y_{1}(y)+Y_{2}(y)=0,(2)
\end{array}\right.
$$

где $z, y$ - обобщенные координаты щетки по вертикальной и горизонтальной осям; $M$ - масса щетки с учетом массы рычага щеткодержателя; $F_{\text {трр}, ~} F_{\text {трк }}-$ сила трения между щеткой и рычагом щеткодержателя и между щеткой и коллектором; $N_{\mathrm{p}}$ - сила нажатия на щетку; $g$ - ускорение свободного падения на поверхности Земли; $\eta$ - одномерная матрица со значениями высот всех коллекторных пластин (профиль коллектора); $F_{\text {тшд1 }}, F_{\text {тщд2 }}$ - силы трения между щеткой и стенками щеткодержателя; $Y_{1}, Y_{2}$ - горизонтальные упругие силы в местах контакта щетки со стенками щеткодержателя.

Приведенная математическая модель механического взаимодействия щетки с коллектором позволяет получить массивы данных со значениями силы воздействия профиля коллектора на щётку $N_{\text {к }}$, изменяющейся в процессе вращения якоря, и построить диаграмму плотности вероятности распределения данной силы по заданным интервалам. 
С учетом механического взаимодействия щетки с коллектором электрокоррозионная составляющая интенсивности износа принимает вид:

$$
\frac{\Delta И_{\mathrm{\kappa}}}{\Delta t}=K_{\mathrm{\kappa}} n^{\frac{3}{4}} I_{\text {щ }}^{\frac{1}{2}}\left(\sum_{j}\left(N_{\mathrm{\kappa} j} P\left(N_{\mathrm{\kappa} j}\right)\right)\right)^{\frac{1}{2}},
$$

где $P\left(N_{\mathrm{\kappa} j}\right)$ - плотность вероятности нахождения зна-

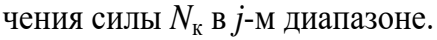

\section{Постановка проблемы}

Известные способы позволяют определить ресурс работы щеток коллекторных электродвигателей только для усредненных показателей режимов работы,

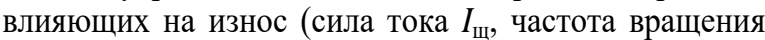
якоря $n$, мощность искрения $P_{\text {и }}$ ) [14-17]. Однако, исходя из представленной выше математической модели процесса изнашивания электрощетки, зависимость ее износа $(\Delta И)$ от силы проходящего по ней тока $\left(I_{\Perp}\right)$ и частоты вращения якоря $(n)$ является нелинейной [14]. Следовательно, при эксплуатации тяговых электродвигателей с варьированием данных показателей с большими отклонениями в широком диапазоне применение усредненных значений показателей $I_{\text {щ }}$ и при моделировании процесса изнашивания неприемлемо.

В настоящее время самосвалы и новый подвижной состав железных дорог оснащаются системами мониторинга показателей режимов работы. Данные системы в режиме реального времени осуществляют измерение и запись широкого набора показателей режимов работы, в число которых у карьерных самосвалов входят: ток якоря, скорость вращения якоря и интенсивность искрения, у локомотивов - ток якоря и скорость движения. При известных параметрах коллекторно-щеточного узла, зная ток якоря, можно рассчитать силу тока $I_{\text {щ, }}$ проходящего по щетке. По известным диаметру колеса, передаточному числу тягового редуктора и скорости движения может быть найдена частота вращения якоря $n$. Интенсивность искрения, измеренная в баллах по ГОСТ 2582-2013 [18], может быть переведена в мощность искрения в относительных единицах. Функциональную зависимость данных величин можно получить после обработки результатов коммутационных испытаний на соответствующем тяговом двигателе с применением прибора контроля коммутации [19, 20].

Таким образом, массивы данных, формируемые системами мониторинга, могут быть применены для формирования прогноза по износу электрощеток с учетом режимов работы тяговых электродвигателей в реальных условиях эксплуатации.

\section{Обработка данных, полученных системой мониторинга параметров режимов работы}

Пример временных диаграмм, полученных системой мониторинга параметров режимов работы, приведен на рис. 3.
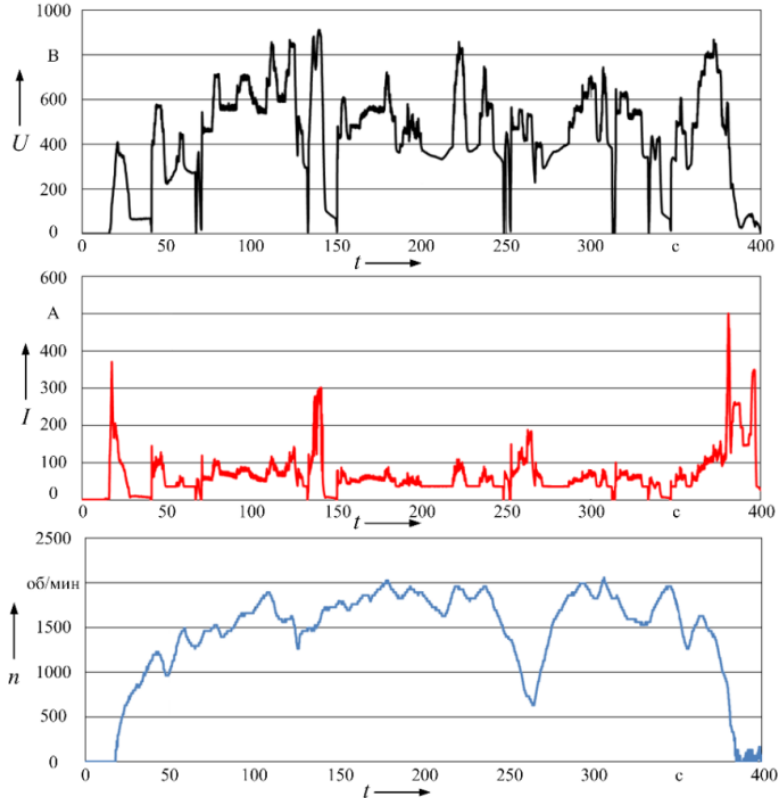

Pис. 3. Временные диаграммы показателей режима работы тягового электродвигателя, полученные бортовой системой мониторинга карьерного самосвала

Fig. 3. Time diagrams of the traction motor operating mode indicators obtained by the on-board monitoring system of the mining dump truck

Массивы данных, полученные с применением бортовых систем мониторинга, позволяют получить диаграммы распределения показателей режимов работы. Примеры полученных распределений мощности искрения под щетками тяговых двигателей карьерных самосвалов приведены на рис. 4.

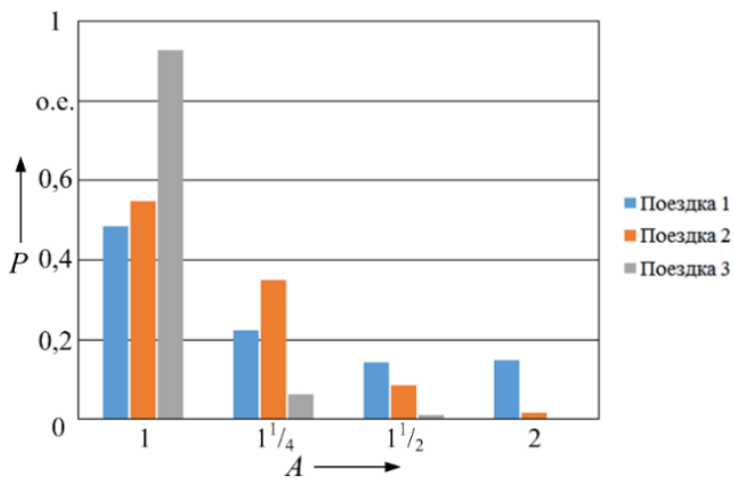

Рис. 4. Распределения интенсивности искрения щеток ТЭД карьерных самосвалов, работающих на различных угольных разрезах

Fig. 4. Distribution of sparking intensity of traction motors brushes of mining dump trucks operating at various coal mines

Примеры распределений показателей режимов работы, фиксируемых системами мониторинга, полученные для тяговых двигателей электровозов, тепловозов и карьерных самосвалов, приведены на рис. 5. 

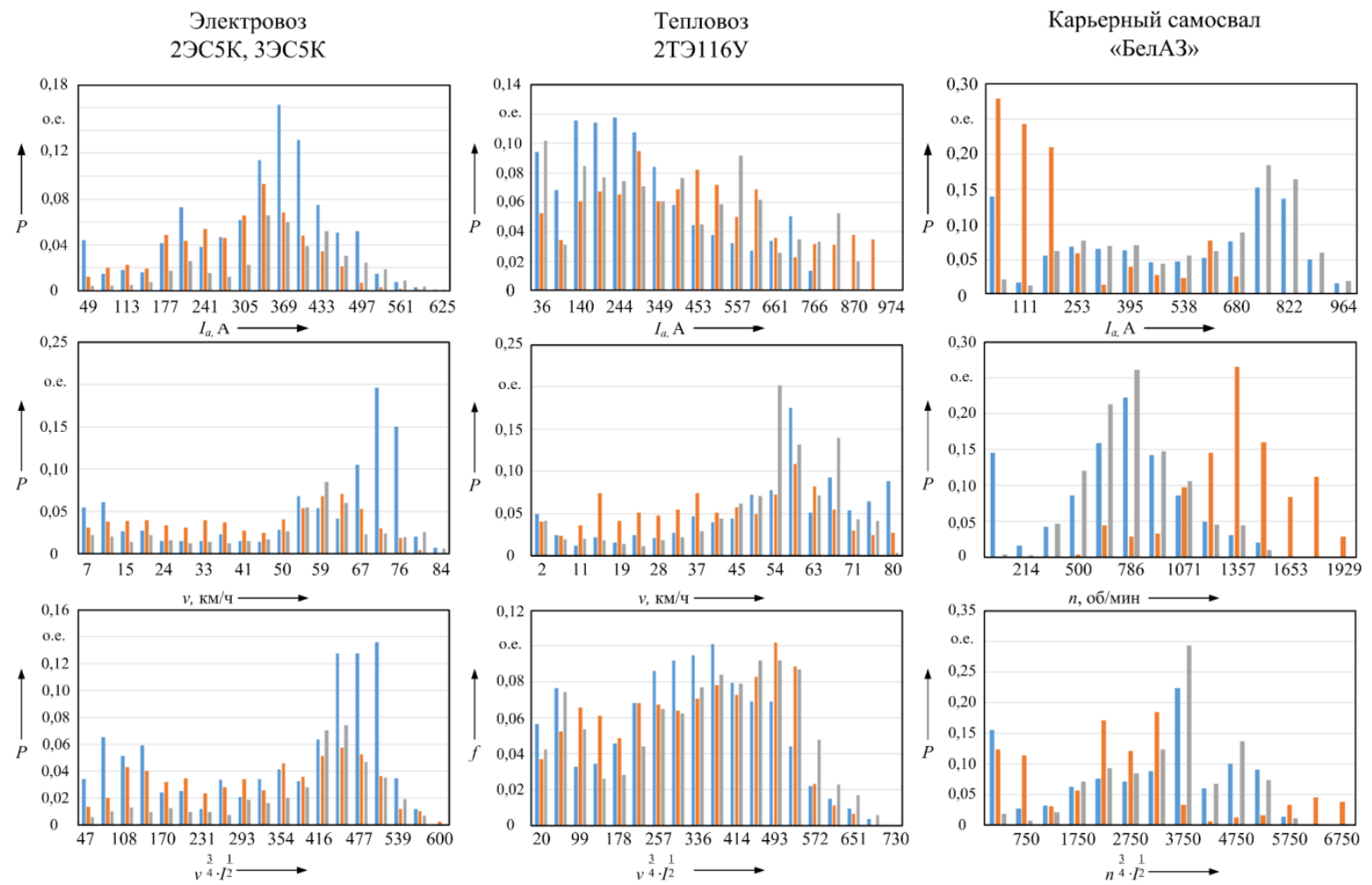

Рис. 5. Распределения показателей режимов работы тяговых электродвигателей электровозов, тепловозов и карьерных самосвалов

Fig. 5. Distributions of indicators of operating modes of traction electric motors of electric locomotives, diesel locomotives and mining dump trucks

\section{Предлагаемый способ прогнозирования остаточного ресурса электрощеток тяговых электродвигателей и алгоритм его реализации}

При наличии обработанных массивов данных, полученных от бортовой системы мониторинга показа- телей режимов работы ТЭД, может быть реализован следующий способ прогнозирования остаточного ресурса электрощеток, иллюстрируемый блок-схемой алгоритма, приведенной на рис. 6.

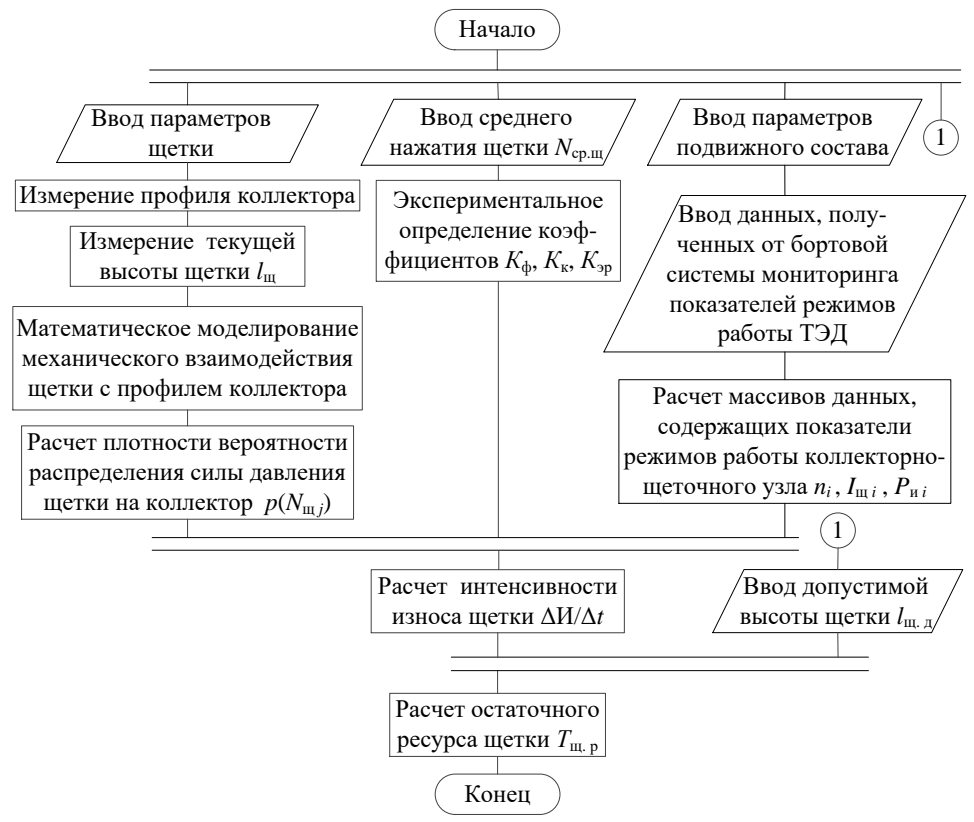

Pис. 6. Блок-схема алгоритма прогнозирования остаточного ресурса электрощеток с учетом массивов данных о показателях режимов работы тягового электродвигателя

Fig. 6. Block diagram of the algorithm for predicting the residual life of electric brushes taking into account the data sets on the indicators of the traction motor operating modes 
В соответствии с приведенной диаграммой для расчета остаточного ресурса электрической щетки тягового электродвигателя требуется определить интенсивность износа щетки как сумму трех составляющих

$$
\frac{\Delta И}{\Delta t}=\frac{\Delta И_{\phi}}{\Delta t}+\frac{\Delta И_{\mathrm{\kappa}}}{\Delta t}+\frac{\Delta И_{\text {эр }}}{\Delta t} .
$$

Для вычисления составляющих интенсивности износа необходимо выполнить три параллельных процесса.

В первом параллельном процессе осуществляется

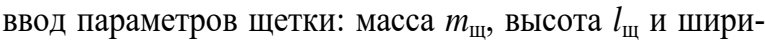
на $b_{\text {щ, }}$ которые могут быть известны из технической документации по электродвигателю либо измерены любыми общеизвестными доступными средствами измерений, имеющими достаточную точность (например, электронные весы и микрометр).

Далее необходимо провести измерение профиля коллектора, т. е. высот коллекторных пластин над произвольно заданным нулевым уровнем, находящемся на некотором расстоянии от оси вращения. Для выполнения этой задачи необходим прибор, позволяющий провести данные измерения (профилометр). Известно множество приборов данного типа с различными принципами действия, например, прибор контроля профиля коллектора машин постоянного тока [20, 21].

На основании известных параметров щетки и профиля коллектора $[22,23]$ выполняется математическое моделирование механического взаимодействия щетки с профилем коллектора [24] в соответствии с системой уравнений (2). Математическое моделирование позволяет найти силу давления щетки на коллектор в дискретно заданное множество моментов времени, следующих друг за другом через равные промежутки [24]. В результате может быть получена плотность вероятности распределения силы давления щетки на коллектор $p\left(N_{\text {щ } j}\right)$.

Во втором параллельном процессе осуществляется ввод среднего нажатия щетки $N_{\text {ср. щ }}$ и определяются значения коэффициентов $K_{\phi}, K_{\mathrm{K}}, K_{\text {эр }}$, которые обусловлены параметрами материала щетки и коллектора и являются постоянными в процессе работы двигателя. Для определения данных коэффициентов проводят три опыта: за равные промежутки времени $\Delta t$ измеряется износ (например, микрометром), обусловленный только фрикционной составляющей, одновременно фрикционной и электрокоррозионной составляющими, сразу тремя составляющими (фрикционной, электрокоррозионной и электроэрозионной).

В первом опыте необходимо без тока обеспечить частоту вращения якоря двигателя $n$ в течение заданного промежутка времени $\Delta t$ при постоянной величине нажатия на щетки $N_{\text {ср.щ. }}$ В данном и следующих опытах для контроля значения частоты вращения якоря необходим тахометр, отсчет промежутка времени $\Delta t$ ввиду его относительно большого значения (порядка десятков часов) может быть произведен, например, с помощью таймера (или обычных бытовых часов), величина нажатия на щетку может быть измерена динамометром. По полученному в опыте значению $\Delta И_{\phi}$ можно определить коэффициент:

$$
K_{\phi}=\frac{n N_{\text {ср.щ }} \Delta t}{\Delta U_{\phi}} .
$$

Во втором опыте необходимо в течение заданного промежутка времени $\Delta t$ при неизменных $N_{\text {ср.щ }}$ и $n$ обеспечить протекание тока $I_{\text {щ }}$ по щетке при отсутствии искрения под щетками. По полученному в опыте значению $\left(\Delta И_{\phi}+\Delta И_{\kappa}\right)$ можно определить коэффициент:

$$
K_{\mathrm{\kappa}}=\frac{\frac{\left(\Delta{И_{\phi}}_{\phi} \Delta И_{\mathrm{\kappa}}\right)}{\Delta t}-K_{\phi} n N_{\text {cp.щ }}}{n^{\frac{3}{4}} I_{\text {щ }}^{\frac{1}{2}} N_{\text {cp.щ }}^{\frac{1}{2}}} .
$$

В третьем опыте необходимо при неизменных $N_{\text {ср.щ }}$ и $n$ обеспечить протекание тока $I_{\text {щ }}$ по щетке при

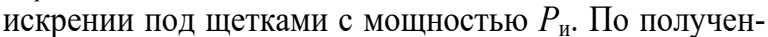
ному в опыте значению $\left(\Delta И_{\phi}+\Delta И_{\kappa}+\Delta И_{э р}\right)$ можно определить коэффициент:

$$
K_{\text {эр }}=\frac{\frac{\left(\Delta И_{\phi}+\Delta И_{\text {к }}+\Delta И_{\text {эр }}\right)}{\Delta t}-K_{\phi} n N_{\text {ср.щ }}-K_{\text {к }} n^{\frac{3}{4}} I_{\text {щ }}^{\frac{1}{2}} N_{\text {ср.щ }}^{\frac{1}{2}}}{n P_{\text {и }}} .
$$

Уровень искрения в третьем опыте задается исследователем путем подпитки или отпитки током добавочных полюсов (что приводит соответственно к усилению или ослаблению их магнитодвижущей силы), к обмотке которых параллельно подключают внешний источник ЭДС (в качестве которого может служить управляемый выпрямитель с возможностью регулирования тока на выходе). При этом необходимо производить объективную оценку мощности искрения $P_{\text {и }}$ под щетками двигателя (в относительных единицах), для чего может быть использовано, например, устройство для измерения интенсивности искрения на коллекторе электрической машины [20] подвижного состава (количество щеток в щеткодержателях, размеры щеток, число параллельных ветвей обмотки якоря, передаточное число тягового редуктора, диаметр качения колесной пары), а также данных, полученных от бортовой системы мониторинга показателей режимов работы ТЭД. Данная система мониторинга должна осуществлять фиксацию текущего времени, токов якоря ТЭД, скорости движения, мощности искрения под щетками (в относительных единицах). Введенные параметры и данные позволяют расчетным путем найти массивы данных, содержащих по

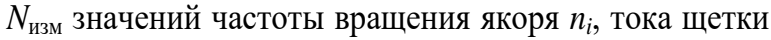
$I_{\text {щ } i}$ и мощности искрения $P_{\text {и } i}$, где $i=1 \ldots N_{\text {изм }}$.

По имеющимся массивам данных производится расчет трех составляющих интенсивности износа щетки, в котором мощность искрения $P_{\text {и }}$ принимают равной среднеарифметическому значению массива элементов $P_{\text {и } i}$.

Определение электроэрозионной составляющей интенсивности износа щетки осуществляется с учетом плотности распределения давления в контакте $p\left(N_{\Perp}\right)$, найденной из математической модели механического взаимодействия щетки с профилем коллектора по выражению (2). 
Расчет фрикционной составляющей интенсивности износа щетки производится по выражению:

$$
\frac{\Delta U_{\phi}}{\Delta t}=K_{\phi} n N_{\text {ср.щ }} \text {. }
$$

Расчет электроэрозионной составляющей интенсивности износа щетки производится по выражению:

$$
\frac{\Delta И_{\text {эр }}}{\Delta t}=K_{\text {эр }} n P_{\text {и }} .
$$

При расчете электроэрозионной составляющей интенсивности износа щетки в выражение (4) необходимо подставить мощность искрения $P_{\text {и }}$, измеренную тем же оборудованием и в тех же единицах измерения, как это осуществлено выше при определении $K_{\text {эр }}$.

При расчете фрикционной и электроэрозионной составляющих интенсивности износа щетки частоту вращения $n$ принимают равной среднеарифметическому значению массива элементов $n_{i}$.

Расчет электрокоррозионной составляющей интенсивности износа щетки выполняют по выражению

$$
\frac{\Delta И_{\mathrm{\kappa}}}{\Delta t}=K_{\mathrm{\kappa}} \frac{1}{N_{\text {изм }}} \sum_{i}\left(n_{i}^{\frac{3}{4}} I_{\text {щ } i}^{\frac{1}{2}}\right)\left(\sum_{j}\left(N_{\text {щ } j} P\left(N_{\text {щ } j}\right)\right)\right)^{\frac{1}{2}} .
$$

Остаточный ресурс работы щетки, выраженный в часах, при усредненных показателях работы ТЭД определяется по выражению (1).

Для апробации разработанного способа прогнозирования остаточного ресурса ТЭД выполнены испытания щеток восьми различных производителей, применяемых на локомотивах 2ТЭ116У. С применением данных, полученных из систем мониторинга локомотивов данного типа, по выражению (3) рассчитаны составляющие износа щеток $\left(\Delta И_{\phi}, \Delta И_{\kappa}, \Delta И_{э р}\right)$, усредненное соотношение которых приведено на рис. 7.

С целью верификации математической модели изнашивания электрощеток, лежащей в основе разработанного способа прогнозирования их остаточного ресурса, выполнено сравнение результатов 31 эксперимента с соответствующей выборкой расчетных данных. Экспериментальные данные получены на установке с двигателем типа 2ПН. Износ определялся гранулометрическим методом $[25,26]$ при варьировании в широком диапазоне тока и частоты вращения

\section{СПИСОК ЛИТЕРАТУРЫ}

1. Статистика // Министерство энергетики Российской Федерации. 2021. URL: https://minenergo.gov.ru/activity/statistic (дата обращения 25.02.2021).

2. Программа развития угольной промышленности России на период до 2035 года (Утверждена распоряжением правительства Российской Федерации от 13.06.2020 № 1582-Р) // Министерство энергетики Российской Федерации. 2020. URL: https://minenergo.gov.ru/node/433 (дата обращения 25.02.2021).

3. Доманов К.И. Анализ непланового ремонта электрического оборудования электровозов постоянного тока в сервисных локомотивных депо // Инновационные проекты и технологии в образовании, промышленности и на транспорте: материалы научной конференции, посвященной Дню российской науки. Омск: ОмГУПС, 2016. - С. 167-173. якоря, а также интенсивности искрения под щетками от 1 до 1⁄2/2 балла. Оценка адекватности математической модели проведена с применением критерия Вилкоксона:

$$
u-M u=u_{\alpha \mathrm{kp}},
$$

где $u$ - полное число инверсий значений выборок; $M u$ - математическое ожидание $u$.

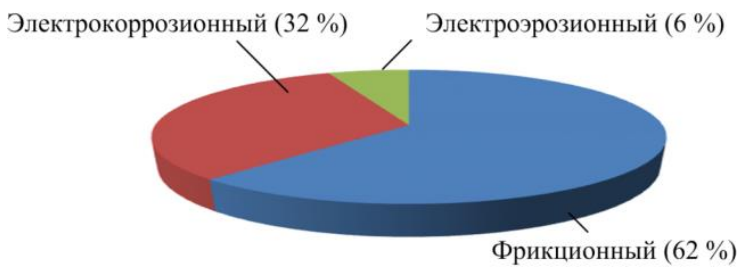

Рис. 7. Соотношение составляющих износа электрощеток

Fig. 7. Proportions of components of wear of electric brushes

Полученные значения: $u=466, M u=480,5$. Для уровня значимости 0,05 табличное значение $u_{\alpha \text { кр }}=139$. Следовательно, различия выборок являются статистически достоверными.

\section{Заключение}

Разработанный способ прогнозирования остаточного ресурса щеток тяговых электродвигателей позволяет учесть показатели режимов работы в условиях реальной эксплуатации и может быть рекомендован для корректировки регламента обслуживания отдельных ТЭД в части введения дополнительного технического обслуживания в случае малого прогнозного значения остаточного ресурса. Алгоритм данного способа может быть применен в бортовых электронных системах подвижного состава для индикации остаточного ресурса электрощеток.

Применение в процессе прогнозирования массивов данных, полученных бортовой системой мониторинга, позволяет повысить точность определения остаточного ресурса электрощеток.

В качестве дальнейших исследований необходимо с целью повышения точности прогнозирования остаточного ресурса электрощеток ТЭД уточнить требуемую периодичность контроля профиля коллектора, обусловленную его изменением в процессе работы.

4. Повышение коммутационной устойчивости тяговых электродвигателей ЭДП-800 карьерных самосвалов / В.В. Харламов, Е.Ю. Чупрына, Р.В. Сергеев, П.К. Шкодун, Д.И. Попов, Ю.В. Москалев // Известия Томского политехнического университета. Инжиниринг георесурсов. - 2018. - Т. 329. - № 7. С. $138-147$.

5. Попов Ю.И. Повышение ресурса тяговых электрических машин электровозов, эксплуатируемых в сложных природноклиматических условиях: автореф. дис. ... канд. техн. наук. Омск, 2018. - 24 с.

6. Kubín J., Ferková Ž., Richter A. Pairing of traction DC motors, long term experiences from practice and simulations // 2019 International Conference on Electrical Drives \& Power Electronics (EDPE). - Slovakia, The High Tatras, 2019. - P. 201-206.

7. ГОСТ 27.310-95. Надежность в технике. Анализ видов, последствий и критичности отказов. Основные положения. - М.: Стандартинформ, 1997. - 18 с. 
8. Izotov A., Timoshenko V., Izotov S. Study of feasibility of controlling tribological situation in sliding current collection assembly of electric machines // 2020 International Conference on Industrial Engineering, Applications and Manufacturing (ICIEAM). - Russia, Sochi, 2020. - P. 1-5.

9. Способ контроля износа щеток и работы щеточноколлекторного узла электрической машины: пат. Рос. Федерация, № 2548020 С2, завял. 05.07.2013; опубл. 10.04.2015. Бюл. № $10 .-10 \mathrm{c}$.

10. Качин О.С., Качин С.И. Увеличение ресурса скользящего контакта электрических машин: монография. - Германия: LAP LAMBERT Academic Publishing, 2012. - 165 c.

11. Способ определения ресурса щеток тяговых коллекторных электродвигателей: пат. Рос. Федерация, № 2677243 С1, завял. 18.07.2017; опубл. 16.01.2019. Бюл. № 2. - $11 \mathrm{c}$.

12. Myshkin N.K., Konchits V.V. Tribology of electrical contacts // Tribologie Und Schmierungstechnik / Eds. Q.J. Wang, Y-W. Chung. - Boston, MA: Springer US, 2015. - V. 64. - P. 5-12.

13. Харламов В.В., Байсадыков М.Ф., Попов Д.И. Методика определения параметров математической модели износа электрических щеток // Инновационные проекты и технологии в образовании, промышленности и на транспорте: материалы научной конференции. - Омск: ОмГУПС, 2017. - С. 109-116.

14. Castevens J.M., Rylander H.G., Eliezer Z. Influence of high velocities and high current densities on the friction and wear behavior of copper-graphite brushes // Wear. - 1978. - V. 49. № 1. - P. 121-130. URL: https://doi.org/10.1016/00431648(78)90142-4 (дата обращения 01.05.2021).

15. Chmelik K., Veselka F. Kluzny kontakt v elektrickych strojich. Ostrava: KEY Publ. s.r.o., 2007. - 256 p.

16. Klopp R.W., Dugnani R., Edmonds J.S. The role of brush spring kinking in a generator flash-over incident // North American Power Symposium. - Boston, MA, 2011. - P. 1-6.

17. Commutation characteristics and brush wear of DC motor at high rotation speed / K. Sawa, M. Isato, T. Ueno, K. Nakano, K. Kondo // 2017 IEEE Holm Conference on Electrical Contacts. - Denver, CO, 2017. P. 176-181.

18. ГОСТ 2582-2013 Машины электрические вращающиеся тяговые. Общие технические условия. - М.: Стандартинформ, 2015. - 102 c.

\section{Информация об авторах}

Харламов B.B., доктор технических наук, заведующий кафедрой электрических машин и общей электротехники Омского государственного университета путей сообщения.

Попов Д.И., кандидат технических наук, доцент кафедры электрических машин и общей электротехники Омского государственного университета путей сообщения.

Байсадыков М.Ф., преподаватель кафедры электрических машин и общей электротехники Омского государственного университета путей сообщения.
19. Sawa K., Ueno T., Nakano K. Evaluation of ARC EROSION of $\mathrm{Cu}$-graphite brush used in small DC motors with and without quenching device // 2020 IEEE 66th Holm Conference on Electrical Contacts and Intensive Course (HLM). - San Antonio, TX, USA, 2020. - P. 170-175.

20. Авилов В.Д. Оптимизация коммутационного процесса в коллекторных электрических машинах постоянного тока: монография. - Омск: Омский научный вестник, 2013. - 355 с.

21. Авилов В.Д., Исмаилов Ш.К. Методика нормирования качества коммутации в тяговых электрических машинах // Известия Транссиба. - 2012. - № 2 (10). - С. 2-6.

22. Eliseev A.V., Eliseev S.V., Orlenko A.I Mathematical modeling of continuous tossing of the brush with the gap from the commutator // International journal of electrical and electronic engineering and telecommunications. - 2019. - V. 2. - P. 72-77. DOI: 10.18178/ijeetc.8.2.72-77

23. Shantarenko S., Ponomarev E., Borodin A. Contact interaction «brush-collector» and working efficiency of locomotive traction electric motor // MATEC Web Conf. - Omsk: OSTU, 2018. V. 239.

24. Харламов В.В., Попов Д.И., Байсадыков М.Ф. Оценка влияния профиля коллектора машины постоянного тока на работу скользящего контакта // Омский научный вестник. - 2016. Вып. 4 (148). - С. 62-65.

25. Забоин В.Н., Веселка Ф. Исследование интенсивности износа электрических щеток методом оптической микроскопии // Механика и физика процессов на поверхности и в контакте твердых тел, деталей технологического и энергетического оборудования: межвузовский сборник научных трудов. Вып. 6. - Тверь: ТвГТУ, 2013. - С. 80-85.

26. Забоин В.Н., Веселка Ф. Новый метод определения износа электрощеток // Мировая наука и образование в условиях современного общества: сборник научных трудов по материалам международной научно-практической конференции. - М.: ООО «АР-Консалт», 2014. - С. 115-118.

Поступила 16.06.2021 г. 


\title{
DEVELOPMENT OF A METHOD FOR PREDICTING THE RESIDUAL LIFE OF ELECTRIC TRACTION MOTOR BRUSHES
}

\author{
Viktor V. Kharlamov1, \\ emoe@omgups.ru \\ Denis I. Popov ${ }^{1}$, \\ emoe@omgups.ru \\ Marsel F. Baysadykov ${ }^{1}$, \\ emoe@omgups.ru \\ 1 Omsk State Transport University,
35, Marks avenue, Omsk, 644046, Russia.
}

The relevance of the research is caused by the need to improve the rolling stock (quarry dump trucks) operation reliability, providing openpit coal mining. This problem is also relevant in the railway transportation industry - on the rolling stock of railways. The reliability of the rolling stock is determined by the reliability of the functioning of its individual components. As it is known from the analysis of failures of rolling stock of vehicles with collector traction motors, one of the critical elements for them is the brush machine. The known methods of forecasting do not fully use the capabilities of modern on-board systems for monitoring the operating modes of traction motors. Therefore, an urgent task is to increase the accuracy of predicting the residual life of electric brushes by taking into account the indicators of the operating modes recorded by the on-board monitoring system.

The main aim of the research is to develop a method for predicting the residual life of electric brushes of traction electric motors of mining dump trucks, taking into account the indicators of operating modes obtained from the on-board monitoring system.

Objects: electric brushes of traction electric motors of mining dump trucks.

Methods: mathematical analysis of data from on-board monitoring systems, mathematical modeling of electric brushes wear, synthesis of a method for predicting the residual life of traction motor brushes.

Results. A method for predicting the residual life of the brushes of traction motors has been developed, which allows taking into account the indicators of the operating modes in real operation conditions. The accuracy of determining the residual life of electrical panels is increased due to the use of data arrays obtained from the on-board monitoring system in forecasting. The authors have developed the prediction algorithm that can be used in on-board electronic systems of rolling stock to indicate the residual life of electric brushes.

\section{Key words:}

Mining dump truck, traction motor, electric brush wear, residual life forecasting, data sets, monitoring system, mathematical modeling.

\section{REFERENCES}

1. Statistika. Ministerstvo energetiki Rossiyskoy federatsii [Statistics. 2021. Ministry of Energy of the Russian Federation]. Available at: https://minenergo.gov.ru/activity/statistic (accessed 25 February 2021).

2. Programma razvitiya ugolnoy promyshlennosti Rossii na period do 2035 goda (Utverzhdena rasporyazheniem pravitelstva Rossiyskoy federatsii ot 13.06.2020 № 1582-P). Ministerstvo energetiki Rossiyskoy federatsii [The Program of development for coal industry of Russia up to 2035 (Approved by Decree of the Government of the Russian Federation from 13.06.2020 № 1582-R). 2020. Ministry of Energy of the Russian Federation]. Available at: https://minenergo gov ru/node/433 (accessed 25 February 2021).

3. Domanov K.I. Analiz neplanovogo remonta elektricheskogo oborudovaniya elektrovozov postoyannogo toka v servisnykh lokomotivnykh depo [Analysis of unscheduled repairs of DC locomotive electrical equipment at locomotive service depots]. Innovatsionnye proekty $i$ tekhnologii v obrazovanii, promyshlennosti $i$ na trans porte: materialy nauchnoy konferentsii, posvyashhennoy Dnyu rossiyskoy nauki [Innovative projects and technologies in education, industry and transport: Proc. of a scientific conference dedicated to Russian Science Day]. Omsk, OSTU Publ., 2016 pp. $167-173$.

4. Kharlamov V.V., Chupryna E.Yu., Sergeev R.V., Shkodun P.K., Popov D.I., Moskalev Yu.V. Enhancement of commutation stability of traction motors EDP800 trucks. Bulletin of the Tomsk Polytechnic University. Geo Assets Engineering, 2018, vol. 329, no. 7, pp. 138-147. In Rus.

5. Popov Yu.V. Povyshenie resursa tyagovykh elektricheskikh mashin elektrovozov ekspluatiruemykh $v$ slozhnykh prirodnoklimaticheskikh usloviyakh. Avtoreferat Diss. Kand. nauk [Improving the service life of electric traction machines of electric locomo- tives operating in harsh natural and climatic conditions. Cand. Diss. Abstract]. Omsk, 2018. 24 p.

6. Kubín J., Ferková Ž., Richter A. Pairing of traction DC motors, long term experiences from practice and simulations. International Conference on Electrical Drives \& Power Electronics (EDPE). Slovakia, The High Tatras, 2019. pp. 201-206.

7. GOST 27.310-95. Nadezhnost v tekhnike. Analiz vidov, posledstviy $i$ kritichnosti otkazov. Osnovnye polozheniya [State Standard 27.310-95. Reliability in engineering. Analysis of types, effects and criticality of failures. Fundamentals]. Moscow, StandardInform Publ., 1997. $18 \mathrm{p}$

8. Izotov A.I., Timoshenko V.N., Izotov S. A. Study of feasibility of controlling tribological situation in sliding current collection assembly of electric machines. International Conference on Industrial Engineering, Applications and Manufacturing. Sochi, Russia, 2020. pp. $1-5$.

9. Malafeev S.I., Tikhonov Yu.V. Sposob kontrolya iznosa shchetok $i$ raboty shchetochno-kollektornogo uzla elektricheskoy mashiny [Method of monitoring brush wear and brush-collector assembly of an electrical machine]. Patent RF, no. 2548020 C2, 2013.

10. Kachin O.S., Kachin S.I. Uvelichenie resursa skolzyashchego kontakta elektricheskikh mashin: monografiya [Increasing sliding contact life of electrical machines: monography]. Germany, LAP LAMBERT Academic Publishing, 2012. 165 p.

11. Kharlamov V.V., Popov D.I., Baysadykov M.F. Sposob opredeleniya resursa shchetok tyagovykh kollektornykh elektrodvigateley [Method for determining the brush life of traction collector motors]. Patent RF, no. 2677243 C1, 2019.

12. Myshkin N.K., Konchits V.V., Braunovich M. The mechanics and tribophysics of electrical contacts. Journal of Friction and Wear, 2015, vol. 36, pp. 454-467. 
13. Kharlamov V.V., Baysadykov M.F., Popov D.I. Metodika opredeleniya parametrov matematicheskoy modeli iznosa elektricheskikh shchetok [Methodology for determining the parameters of a mathematical model for electrical brush wear]. Innovatsionnye proekty $i$ tekhnologii $v$ obrazovanii, promyshlennosti i na trans porte. Materialy nauchnoy konferentsii [Innovative projects and technologies in education, industry and transport. Proc. of a scientific conference]. Omsk, OSTU Publ., 2017. pp. 109-116.

14. Castevens J.M., Rylander H.G., Eliezer Z. Influence of high velocities and high current densities on the friction and wear behavior of copper-graphite brushes. Wear, 1978, vol. 49, no. 1, pp. 121-130.

15. Chmelik K., Veselka F. Kluzny kontakt $v$ elektrickych stroyich. Ostrava, KEY Publ. s. r. o., 2007. 256 p.

16. Klopp R.W., Dugnani R., Edmonds J.S. The role of brush spring kinking in a generator flash-over incident. North American Power Symposium. Boston, MA, 2011. pp. 1-6.

17. Sawa K., Isato M., Ueno T., Nakano K., Kondo K. Commutation characteristics and brush wear of DC motor at high rotation speed. IEEE Holm Conference on Electrical Contacts. Denver, CO 2017. pp. $176-181$

18. GOST 2582-2013. Mashiny elektricheskie vrashchayushchiesya tyagovye [State Standard 2582-2013. Electric rotating traction machines. General specifications]. Moscow, StandardInform Publ., 2015. $102 \mathrm{p}$.

19. Sawa K., Ueno T., Nakano T. Evaluation of ARC EROsion of $\mathrm{Cu}-$ graphite brush used in small DC motors with and without quenching device. IEEE $66^{\text {th }}$ Holm Conference on Electrical Contacts and Intensive Course (HLM). San Antonio, TX, USA, 2020. pp. $170-175$.

20. Avilov V.D. Optimizatsiva kommutatsionnogo protsessa v kollektornykh elektricheskikh mashinakh postoyannogo toka: monografija [Optimisation of the commutation process in collector-type DC electric machines: monography]. Omsk, Omsky nauchny vestnik Publ., 2013. 355 p.

21. Avilov V.D., Ismailov S.K. Metodika normirovaniya kachestva kommutatsii v tyagovykh elektricheskikh mashinakh [Methodolo- gy for rationing the quality of switching in traction electric machines]. Izvestiya Transsiba, 2012, no. 2 (10), pp. 2-6.

22. Eliseev A.V., Eliseev S.V., Orlenko A.I. Mathematical modeling of continuous tossing of the brush with the gap from the commutator. International journal of electrical and electronic engineering and telecommunications, 2019, vol. 2, pp. 72-77.

23. Shantarenko S.G., Ponomarev E.V., Borodin A.V. Contact interaction «brush-collector» and working efficiency of locomotive traction electric motor. MATEC Web Conference, Omsk, OSTU, 2018. Vol. 239.

24. Kharlamov V.V., Popov D.I., Baysadykov M.F. Otsenka vliyanija profilya kollektora mashiny postoyannogo toka na rabotu skolzyashchego kontakta [Evaluating the effect of DC machine collector profile on the operation of the sliding contact]. Omskiy nauchniy vestnik, 2016, no. 4 (148), pp. 62-65.

25. Zaboin V.N., Veselka F. Issledovanie intensivnosti iznosa elektricheskikh shchetok metodom opticheskoy mikroskopii [Research of the wear intensity in electric brushes by optical microscopy]. Mekhanika i fizika protsessov na poverkhnosti i $v$ kontakte tverdykh tel, detaley tekhnologicheskogo i energeticheskogo oborudovaniya. Mezhvuzovskiy sbornik nauchnykh trudov. Vyp. 6 [Mechanics and physics of processes on the surface and in the contact of solids, parts of technological and power equipment. Interuniversity collection of scientific papers. Iss. 6]. Tver, TSTU Publ., 2013. pp. 80-85.

26. Zaboin V.N., Veselka F. Novy metod opredeleniya iznosa elektroshchetok [New method for determining the wear of electric brushes]. Mirovaya nauka i obrazovanie v usloviyakh sovremennogo obshchestva. Sbornik nauchnykh trudov po materialam mezhdunarodnoy nauchno-prakticheskoy konferentsii [World science and education in modern society. Collection of scientific papers on the materials of the international scientific-practical conference]. Moscow, 2014. pp. 80-85.

Received: 16 June 2021.

\section{Information about the authors}

Viktor V. Kharlamov, Dr. Sc., head of the department, Omsk State Transport University.

Denis I. Popov, Cand. Sc., associate professor, Omsk State Transport University.

Marsel F. Baysadykov, lecturer, Omsk State Transport University. 